\title{
X-ray Mapping Characterisation of Hybrid Alkaline Cement Discolouration Area
}

\section{Qingtao Huang, Zhong Tao, Zhu Pan, Md Kamrul Hassan, Laurel George and Richard Wuhrer}

Western Sydney University, Penrith, New South Wales, Australia

In recent years, due to global warming concerns, the scientific community and industry have become increasingly interested in developing cements with low carbon dioxide $\left(\mathrm{CO}_{2}\right)$ emissions. In particular, alkali-activated hybrid cement, also known as blend cement, has attracted considerable attention [1]. In hybrid cementitious systems, blends of ordinary Portland cement (OPC), fly ash and ground granulated blast-furnace slag (GGBFS) are the most frequently studied [2-4]. The use of fly ash significantly reduces $\mathrm{CO}_{2}$ emissions associated with cement production, while the presence of GGBFS eliminates the need for external heat. The presence of OPC reduces the amount of alkaline activator, allowing it to be made available on an industrial scale $[5,6]$.

These cement types can be prone to discolouration issues however, found mostly at the cement surface. In order to discover the reason for discolouration issues, X-ray mapping (XRM) has been used on a sample of alkali-activated hybrid cement, along with X-ray diffraction (XRD) and Fourier-transform infrared spectroscopy (FTIR). In the example given here, discolouration was found on the surface of the sample, with the colour gradually changing from a darker grey, to a lighter grey/yellow over time (Figure 1a-c). This interesting phenomenon suggests that there may be a change in composition and microstructure. XRD analysis of the surface compared to the internal regions of the sample did not show obvious signs of phase change, except for intensity changes of silicon oxide and calcite. FTIR analysis showed possible $\mathrm{CH}_{3} / \mathrm{CH}_{2}$ stretching $\left(2901,2988 \mathrm{~cm}^{-1}\right)$ in the internal region of the cement sample, and also revealed differences in the $\mathrm{Si}-\mathrm{O}$ stretching $\left(960,1046 \mathrm{~cm}^{-1}\right)$ between the internal and surface regions of the sample, agreeing with XRD data.

To obtain a better understanding of how the elemental composition of the sample was changing, XRM was then utilized (Figure 1d-k). XRM is an excellent tool for characterising the distribution of elements and phases in a cement sample [7]. Further to this, the use of colour can increase the visibility of intensity levels in the map [8] and reveal variations more easily. Pseudo colouring was used here to distinguish between different elements in the cement sample. The colours red, green and blue were assigned to silicon ( $\mathrm{Si}$ ), sulphur $(\mathrm{S})$ and potassium $(\mathrm{K})$ respectively (Figure $2 \mathrm{~b}$ ). The resulting map indicated the presence of a highly concentrated sulphur and potassium phase (light blue in the map) at the interface of the internal and surface regions. A subsequent potassium/sulphur ratio map was created (Figure 2c) showing how the ratio of these two elements changes with sample depth. In this map darker areas represent higher concentrations of sulphur, which can be seen at the interface of the surface and interior region. This suggests these two elements have migrated during the drying of the cement sample, which may be the cause of the discolouration. The surface shows there is less sulphur compared to potassium.

\section{Acknowledgements}

The authors wish to thank Western Sydney University (WSU), Centre for Infrastructure Engineering (CIE) and the Advanced Materials Characterisation Facility (AMCF) 


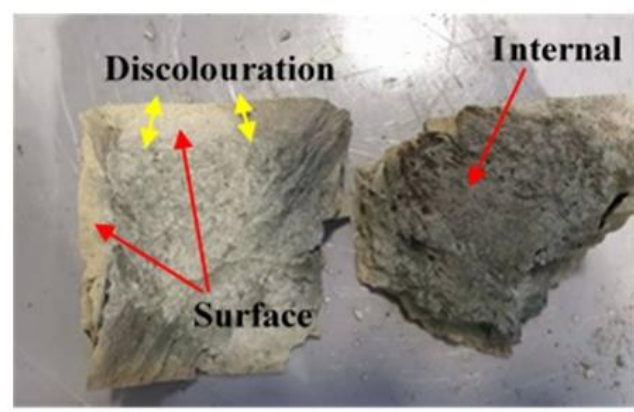

a.

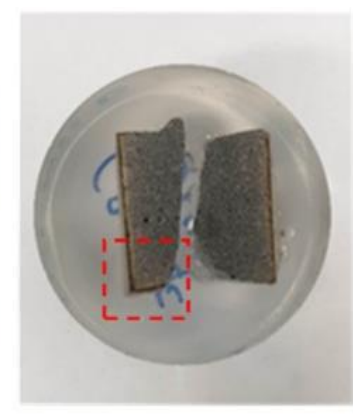

b.

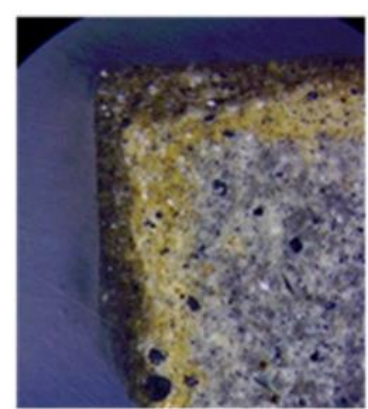

c.
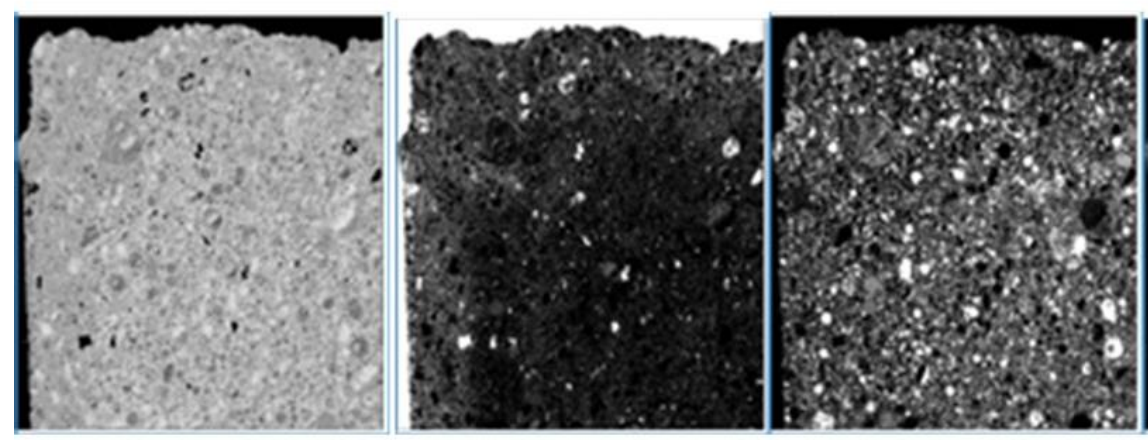

d.

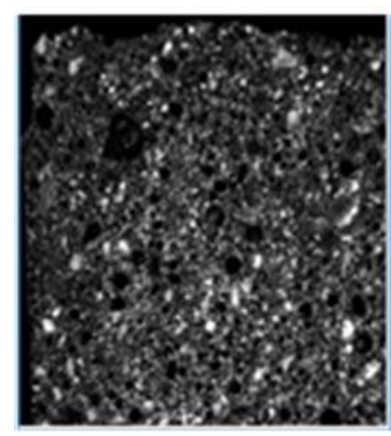

h. e.

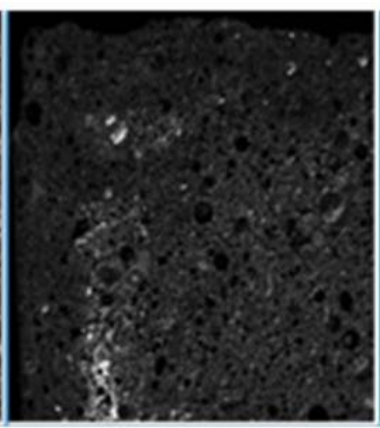

i. f.

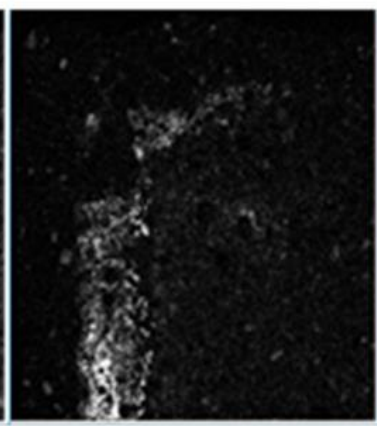

j.

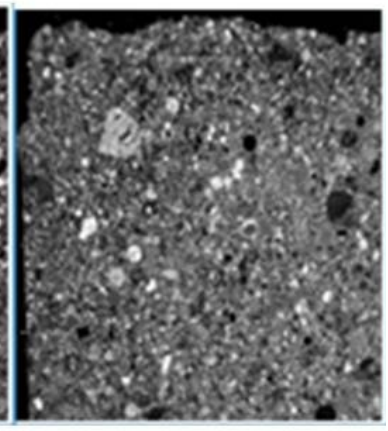

g.

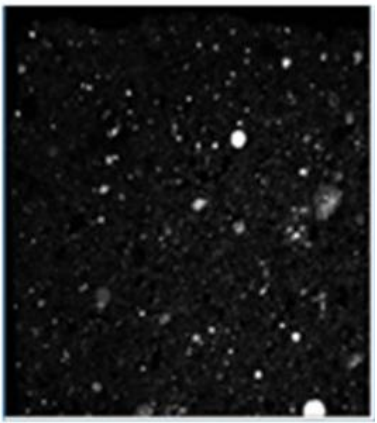

$\mathrm{k}$.

Figure 1. Alkali-activated fly ash hybrid cement, showing a) an image of crushed cement sample shows discoloration between the surface and internal area, b) an image of cement pieces mounted in epoxy resin and polished, and c) an optical microscope image of the area for $\mathrm{x}$-ray mapping. Images of the interface between surface and internal with varying concentration range. d) SEI Image, e) carbon, f) aluminium, g) silicon, h) calcium, i) potassium, j) sulphur and k) iron. Maps collected at 15keV, 1024x1024 pixel, 50 mesc/pixel. Horizontal width of field $(\mathrm{HWOF})=1.12 \mathrm{~mm}$. 


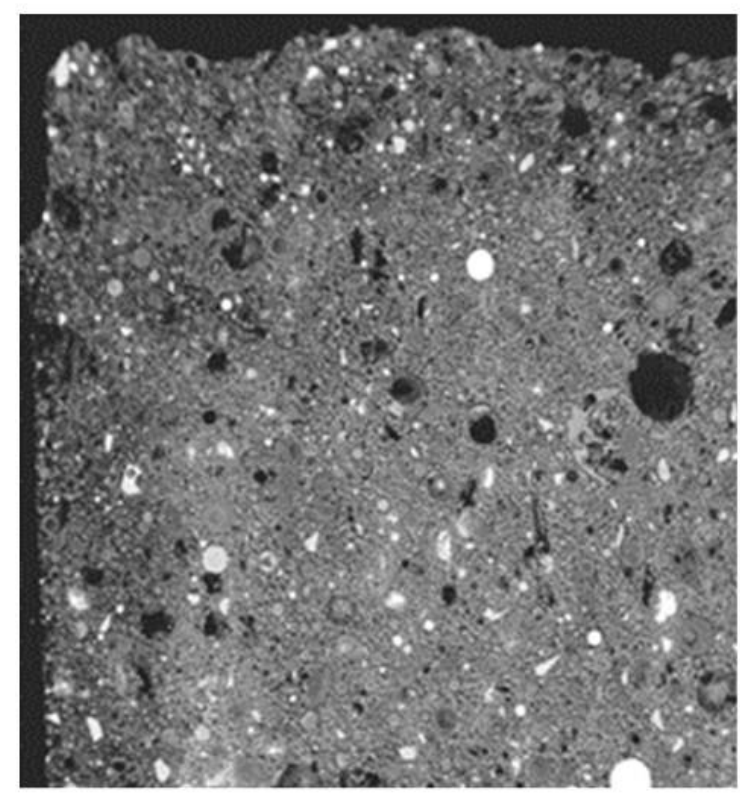

a.

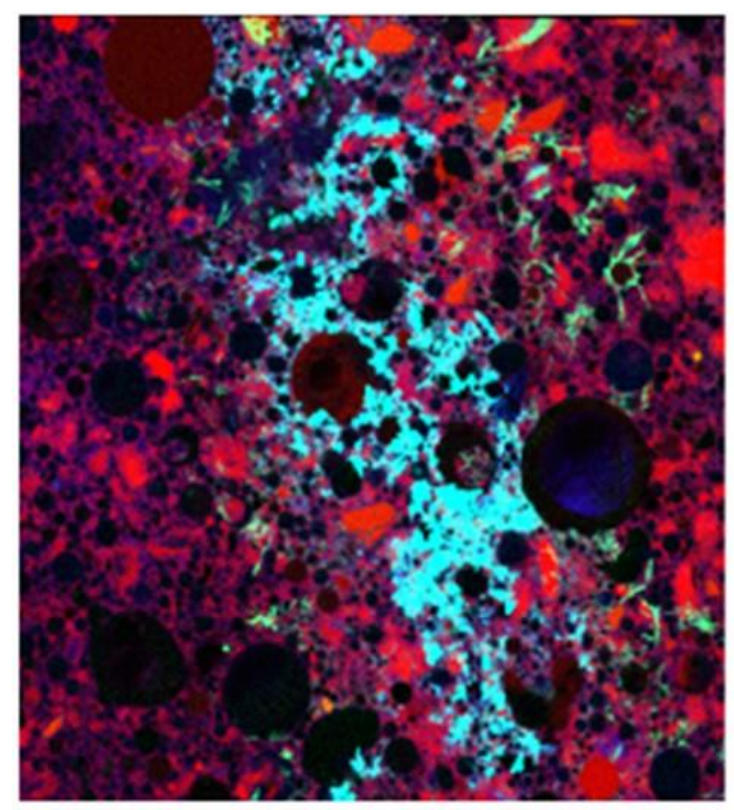

c.

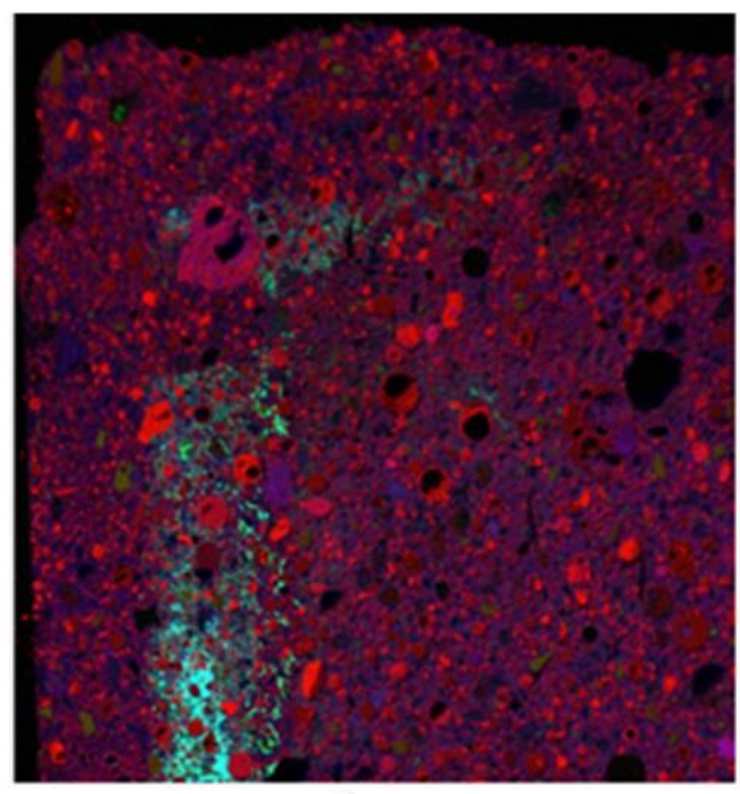

b.

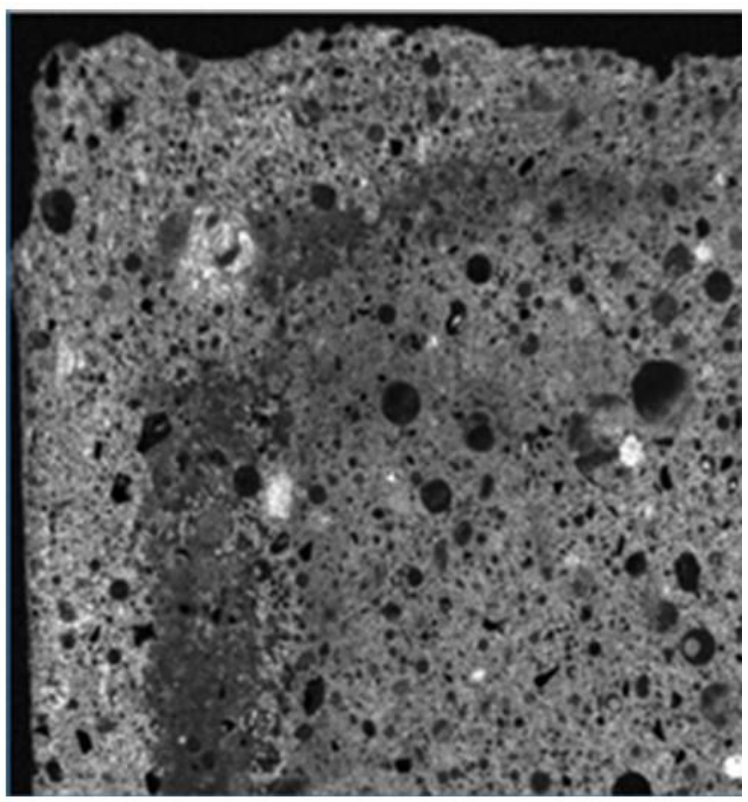

d.

Figure 2. XRM of the discoloured area showing a) BSE image, b) pseudo colour image of the interface (red=silicon, green=sulphur, blue=potassium), c) a magnified view of the interface showing a high concentration of potassium and sulphur present in the interface, and d) a potassium/sulphur ratio map, where dark areas represent a higher sulphur concentration.

\section{References}

1. Prud'homme, E., E. Joussein, and S. Rossignol, Handbook of Alkali-Activated Cements, Mortars and Concretes, ed. F. Pacheco-Torgal, et al. 2015, Oxford: Woodhead Publishing. 687-728.

2. Garcia-Lodeiro, I., et al., Hydration of hybrid alkaline cement containing a very large proportion of fly ash: A descriptive model. Materials, 2016. 9(7): p. 605. 
3. García-Lodeiro, I., A. Fernández-Jiménez, and A. Palomo, Variation in hybrid cements over time. Alkaline activation of fly ash-portland cement blends. Cement and Concrete Research, 2013. 52: p. 112122.

4. Palomo, A., et al., Hybrid binders: A journey from the past to a sustainable future (opus caementicium futurum). Cement and Concrete Research, 2019. 124: p. 105829.

5. Li, Z. and S. Liu, Influence of slag as additive on compressive strength of fly ash-based geopolymer. Journal of Materials in civil engineering, 2007. 19(6): p. 470-474.

6. Askarian, M., et al., Mechanical Properties of Ambient Cured One-part Hybrid OPC-geopolymer Concrete. Construction and Building Materials, 2018.

7. Wuhrer, R., Moran, K., and Phillips, M. R., X-ray mapping and post processing. Microscopy and Microanalysis, 2006. 12(S2).

8. Wuhrer, R., and Moran, K., X-ray mapping characterisation of materials that have a large dynamic compositional range. Microscopy and Microanalysis, 2016. 22(S3). 